\title{
Le tuberculome intramédullaire : une rare cause infectieuse de compression médullaire
}

\section{Intramedullary tuberculoma: a rare infectious cause of spinal cord compression}

\author{
Diallo $\mathrm{M}^{1}$, Agaly $\mathrm{H}^{1}$, Kourouma $\mathrm{D}^{1}$, Sogoba $\mathrm{Y}^{1}$, Sogoba B ${ }^{1}$, Diallo $\mathrm{O}^{2}$, Kanikomo $\mathrm{D}^{1}$ \\ 1-Service de neurochirurgie CHU Gabriel Touré \\ 2-Service de neurochirurgie CHU hôpital du Mali
}

Auteur correspondant: Dr Diallo Moussa CHU Gabriel Touré Bamako, BP : 267

e-mail:mdilallo5@gmail.com

\section{Résumé}

Le tuberculome est l'une des rares causes infectieuses de compression médullaire. Il est responsable de troubles neurologiques importants avec des séquelles considérables. L'objectif était de discuter des aspects physiopathologiques et la conduite thérapeutique du tuberculome intramédullaire. Patient et méthode : Le patient de 32 ans sans antécédents particuliers a présenté un syndrome de compression médullaire lente. La chirurgie associée à l'examen anatomopathologique ont permis de poser le diagnostic. Une antibiothérapie antituberculeuse a été instaurée en traitement de fond. Après 15 mois, l'évolution était marquée par la persistance des troubles neurologiques. Conclusion : La prise en charge précoce et adéquate du tuberculome intramédullaire limite le risque de complication mais ne garantis pas une évolution sans séquelles.

Mots clé : antibiothérapie ; chirurgie ; tuberculome intramédullaire

\section{Summary}

Tuberculoma is one of the rare infectious causes of spinal cord compression. He is responsible for major neurological disorders with considerable sequelae. The objective was to discuss the physiopathological aspects and the therapeutic management of intramedullary tuberculoma. Patient and method: The 32-year-old patient with no specific history presented with slow spinal cord compression syndrome. Surgery combined with pathological examination made it possible to make the diagnosis. Antituberculosis antibiotic therapy was started as a background treatment. After 15 months, the evolution was marked by the persistence of neurological disorders. Conclusion: The early and adequate management of intramedullary tuberculoma limits the risk of complications but does not guarantee an outcome without sequelae.

Keyword: antibiotherapy; intramedullary tuberculoma; surgery

\section{Introduction}

Le tuberculome est cette lésion caractérisée par la formation d'une masse tumorale dont le Mycobactérium Tuberculosis est à la base. Lorsque cette lésion tumorale se développe à l'intérieur de la moelle spinale, on parle de tuberculome intramédullaire. Il s'agit d'une affection rare [1,2] mais grave car elle est responsable de trouble neurologique important parfois irréversible. Le diagnostic de cette maladie n'est pas facile. Les traitements proposés ne sont efficaces que si la prise en charge est précoce. Cette efficacité thérapeutique n'est pas dénuée de séquelles neurologiques. Les auteurs rapportent le cas d'une compression médullaire thoracique par un tuberculome intramédullaire chez un sujet jeune immunocompétent. A travers cette observation, nous allons discuter de la physiopathologie et du traitement de cette affection.

\section{Cas clinique}

L'histoire de ce jeune enseignant de 32 ans sans antécédent a débuté il y a 8 mois de son hospitalisation par l'installation progressive d'un trouble de la marche. Celui-ci était marqué par une fatigabilité des membres inférieurs lors des activités de marche. Aussi, il rapporte une oppression thoracique décrite comme «une sensation d'étau qui serre ». La persistance de ces symptômes l'a amené à consulter un médecin généraliste qui le met sous un traitement antalgique. L'évolution 3 semaines plus tard a été marquée par l'aggravation du trouble de la marche; le patient se déplaçait à l'aide d'une canne. Il fut conduit par sa famille chez un tradi-praticien où il a reçu un traitement à base de décoctions. La survenue d'une incontinence urinaire et d'une aggravation de la faiblesse aux membres inférieurs ont nécessité son transfert à l'hôpital. L'examen à son arrivée a mis en évidence un déficit moteur aux membres inférieurs avec une 
force motrice cotée à $2 / 5$ de façon bilatérale. Les réflexes ostéotendineux étaient vifs en rotulien et en achilléen. Le signe de Babinski était présent et bilatéral. On notait une névralgie intercostale circonscrivant le thorax en une ceinture passant par les mamelons. L'examen de la sensibilité avait trouvé une hypoesthésie en bande allant des mamelons à l'appendice xiphoïde et une anesthésie en dessous jusqu'aux orteils. Une incontinence urinaire accompagnait le tableau clinique. Il n'y avait pas de syndrome rachidien (douleurs et déformations). La température était à $37,4^{\circ} \mathrm{C}$, la pression artérielle à $125 / 80 \mathrm{~mm} \quad \mathrm{Hg}$. A l'auscultation, les bruits du cœur étaient réguliers et les poumons libres. L'imagerie par résonance magnétique (IRM) réalisée avait permis de mettre en évidence en séquence T2 sans injection de Gadolinium, une lésion intramédullaire hypointense en regard des vertèbres thoraciques T3-T4 associée à un œdème intramédullaire étendu (Figure 1 A et B). L'injection de Gadolinium en séquence $\mathrm{T} 1$ a révélé une lésion multi nodulaire avec prise de contraste en périphérie (Figure $1 \mathrm{C}$ et D).
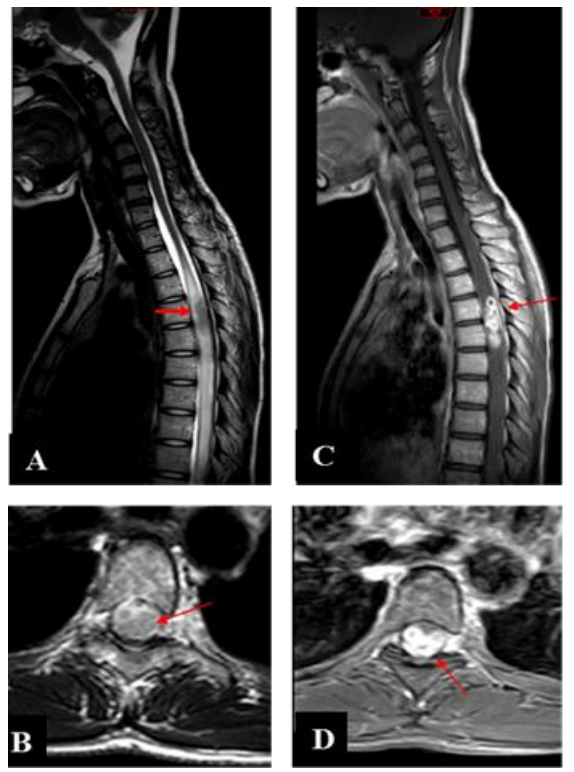

Figure 1 : IRM Médulaire

(A) coupe sagittale séquence T2 : Lésion hypointense en regard des vertèbres T3-T4.

(B) coupe axiale séquence T2 : Lésion hypointense occupant les 2/3 du canal.

(C) coupe sagittale séquence T1 injectée: Lésion multinodulaire avec prise de contraste.

(D) coupe axiale séquence T1 injectée : Lésion hypointense avec prise de contraste.

La tomodensitométrie thoraco-abdomino-pelvienne (Scan TAP) à la recherche d'étiologie de cette compression intramédullaire n'avait pas trouvé de lésion primitive (Figure $2 \mathrm{E}$ et $\mathrm{F}$ ).
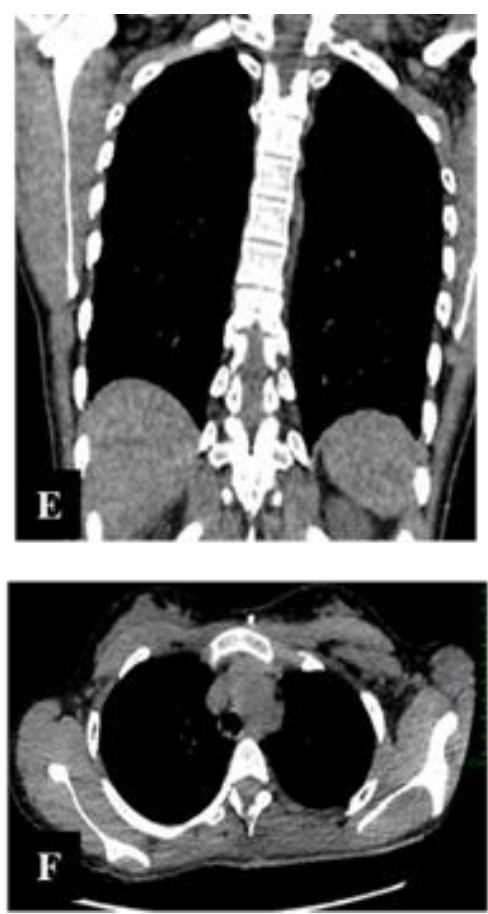

Figure 2 : Tomodensitométrie thoracique (E) coupe axiale; $(\mathrm{F})$ coupe coronale ne montrant pas d'image en faveur d'une tuberculose pulmonaire

L'indication d'une exérèse chirurgicale de la lésion intramédullaire est posée. Au bilan préopératoire le taux d'hémoglobine était à $12,8 \mathrm{~g} / \mathrm{dl}$, l'hématocrite à $37,7 \%$, les plaquettes à $17910^{3} \mu \mathrm{L}$, les globules blancs $8000 / \mathrm{mm}^{3}$. La Protéine $\mathrm{C}$ réactive (CRP) était à 7. Le patient était de groupe sanguin $\mathrm{B}$ rhésus positif. Après une laminectomie des vertèbres T3, T4 et T5, l'ouverture de la dure-mère avait permis d'observer un aspect de «grosse moelle ». La myélotomie donne accès à une lésion légèrement hypodense enchassée dans la moelle rendant difficile une ablation complète. Une exérèse partielle de la lésion a été réalisée. L'examen anatomopathologique de la pièce opératoire a conclu à un tuberculome intramédullaire active. En postopératoire immédiat, le patient a présenté une détérioration du déficit neurologique avec une force musculaire passée à $0 / 5$ aux membres inférieurs. Une corticothérapie à base Méthylprednisolone à la dose $80 \mathrm{mg}$ a été administrée par voie veineuse pendant 3 jours. Un traitement de fond de 13 mois a été mis en route. Il s'agissait d'une association Rifampicine $600 \mathrm{mg} / \mathrm{j}+$ Isoniazide $150 \mathrm{mg} /$ + Pyrazinamide $1000 \mathrm{mg} / \mathrm{J}$ pour 3 mois et puis de la Rifampicine $600 \mathrm{mg}+$ Isoniazide $300 \mathrm{mg}$ pour 10 mois (3RHZE $+10 \mathrm{RH})$. La kinésithérapie motrice des membres inférieurs et la rééducation sphinctérienne complétaient la prise en charge. A deux mois postopératoire, la névralgie intercostale avait cessé sous Lyrica ${ }^{\circledR} 50 \mathrm{mg}$ à la dose de 2 comprimés par jours. La force motrice aux membres inférieurs était revenue à $2 / 5$. Le patient était toujours sous sonde urinaire, A 9 mois d'évolution le déficit était coté à $3+/ 5$, la marche était possible avec l'aide d'un déambulateur. Le 
patient signalait une impériosité mictionnelle. Quinze mois après la chirurgie, la force motrice à $4+/ 5$ autorisait la marche avec l'aide d'une canne. Le patient portait une protection (couche) pour des fuites mictionnelles intermittentes.

\section{Discussion}

Le tuberculome intramédullaire est une affection rare. Sa prévalence varie entre 1 et $2 / 100000$ patients porteurs de tuberculose [1,2]. Il serait légèrement prédominant chez la femme [3] (Tableau I). L'absence de signes d'imprégnation tuberculeuse et de foyer primitif de tuberculose chez notre jeune patient immunocompétent constitue une particularité qui mérite d'être souligné. Plusieurs auteurs s'accordent à dire que l'atteinte médullaire se fait généralement par voie hématogène à partir d'un foyer tuberculeux développé à distance dans l'organisme [4,5]. Habituellement, il s'agit d'une tuberculose pulmonaire évolutive [3-8]. Dans le cas présent l'atteint est isolé avec une absence de lésion pulmonaire (Figure 2 E-F). Ce qui laisserait supposer une autre physiopathologie de la maladie. Il n'y a pas de tableau clinique spécifique au tuberculome intramédullaire. La présentation clinique est celle d'une compression médullaire lente par une tumeur intramédullaire. Tout comme chez notre patient, le syndrome infectieux peut cruellement manquer au tableau clinique égarant ainsi le diagnostic. L'IRM médullaire est l'examen de choix au diagnostic de la compression médullaire quelle que soit la cause. Elle a une sensibilité et une spécificité supérieures au scanner pour le diagnostic des lésions du système nerveux. L'accessibilité à cet examen demeure restreinte dans nos pays à cause de son coût et de sa disponibilité. A défaut de l'IRM, le myéloscanner peut servir d'examen de débrouillage au diagnostic de la lésion de compression médullaire. Le siège intramédullaire thoracique semble être le plus fréquent [6] (Tableau I) même si une localisation au niveau du cône terminal a été rapportée [7]. Le traitement du tuberculome intramédullaire est difficile [6] sans doute à cause de l'absence de consensus. Des cas de récupération complète sous traitement antibiotique et sans la chirurgie ont été rapportés dans la littérature $[9,10]$. Les molécules utilisées et la durée du traitement sont variables d'une étude à l'autre. Tout comme dans notre observation, d'autres auteurs avaient eu recours à la corticothérapie pour son effet antiinflammatoire $[8,9,10$,$] . La chirurgie est l'option thérapeutique la$ plus rependue $[3,6,7,9,10]$. Chez notre patient, l'exérèse a été partielle car la lésion était adhérente et il n'y avait pas de plan de clivage franc avec le tissu médullaire sain. L'examen anatomopathologique est la clé de voute du diagnostic. L'association d'antibiotiques antituberculeux est le traitement de fond qui vient en complément de la chirurgie. Elle permet d'obtenir la guérison lorsqu'elle est efficace. La prise en charge précoce et optimale permet de réduire les risques de séquelles neurologiques. Après 15 mois d'évolution, notre patient présentait des troubles neurologiques résiduels. Il s'agissait d'une impériosité mictionnelle et d'une paraparésie.

\section{Conclusion}

Le tuberculome intramédullaire est une affection rare. Cette rareté ne doit pas faire méconnaitre sa gravité car elle est responsable de handicap moteur parfois important et irréversible. Le jeune âge, l'immunocompétence et l'absence de signes d'imprégnation tuberculeuse ne constituent pas des éléments permettant d'écarter le diagnostic. La prise en charge précoce et optimale chez un sujet jeune permet d'espérer un résultat favorable. L'antibiothérapie antituberculeuse constitue le traitement de fond qui garantit la guérison.

Conflit d'intérêt : aucun

\section{Références}

1. Citow JS, Ammirati M. Intramedullary tuberculoma of the spinal cord:case report. Neurosurg. 1994;35:327-30.

2.Liu J, Zhang $\mathrm{H}$, He B, et al. Intramedullary tuberculoma combined with abscess: case report and literature review. World Neurosurg. 2016;89:726.e1-4.

3.Li H, Liu W, You C. Central nervous system tuberculoma. Journal of Clinical Neuroscience 2012;19:691-695.

4.Hoda MF, Prasad R, Singh V.P, Maurya P. Spinal intramedullary tubercular abcess. Indian $\mathrm{J}$ Tuberc 2005; 52:211-214.

5.Jaiswal AK, Jaiswal S, Gupta SK, Singh Gautam VK, Kumar S. Intramedullary tuberculoma of the conus. J ClinNeurosci 2006;13:870-2.

6.Ben Salah A, Hachicha A, Kolsi F, Kherifech M, Kammoun B, Boudawara MZ. Tuberculome intramédullaire : à propos d'un cas et revue de la littérature. Neurochirurgie 64 (2018) 240-277.

7.Rivière F, Staub E, Le Floch $\mathrm{H}$ et al. Diagnostic et traitement d'un tuberculome intramédullaire : quelle place pour la neurochirurgie ? Presse Med. 2011; 40: 314-323.

8.Wang GC, Wu SW. Spinal intramedullary tuberculoma following pulmonary tuberculosis: A case report and literature review. Medicine (Baltimore). 2017 Dec; 96(49): e8673.

9.Jaiswal M, Gandhi A, Sharma A, Mittal RS. Experiences and Conceptualisation of Spinal Intramedullary TuberculomaManagement.Korean $\mathbf{J}$ Spine. 2015 Mar; 12(1): 5-11.

10.Varghese P, Abdul Jalal MJ, KandathilJC,Mathew IL, Spinal Intramedullary Tuberculosis. Surg J. 2017 Apr; 3(2): e53-e57. 